\title{
Efeito do Envelhecimento nas Propriedades Mecânicas e Dinâmico-Mecânicas de Composições de Borracha Natural com Mica
}

\author{
Viviane A. Escócio, Agnes F. Martins, Leila L. Y. Visconte, Regina C. R. Nunes
}

IMA, UFRJ

\begin{abstract}
Resumo: O estudo do efeito do envelhecimento em artefatos é principalmente importante quanto ao custo e qualidade, tornando o material atrativo ou não para produtos expostos a condições climáticas mais exigentes. Neste trabalho foi estudado o efeito do envelhecimento acelerado em composições vulcanizáveis de borracha natural com mica. $\mathrm{O}$ teor da carga mineral variou entre 0 e $40 \mathrm{phr}$. As diferentes composições foram avaliadas, antes e após envelhecimento, quanto às propriedades mecânicas e dinâmico-mecânicas, e também quanto à densidade de ligações cruzadas. Todos os dados foram comparados aos da composição sem carga. Os resultados mostraram que o teor de mica na formulação estudada, antes e após envelhecimento, não deve ser superior a 30phr. Pôde também ser concluído que as características da mica foram as responsáveis pela resistência ao envelhecimento das composições de borracha natural, nas condições escolhidas. As propriedades dinâmico-mecânicas, obtidas por DMTA, corroboraram as propriedades mecânicas avaliadas.
\end{abstract}

Palavras-chave: Borracha natural, mica, propriedades fisico-mecânicas, propriedades dinâmico-mecânicas, envelhecimento.

\section{Effect of Ageing on Mechanical and Dynamic Mechanical Properties of Natural Rubber Compositions with Mica}

\begin{abstract}
The effects from ageing of materials is important in terms of cost and quality, particularly if the products are to be used under severe weather conditions. In this work, the effect of accelerated ageing in vulcanizable compositions of natural rubber and mica was studied. The amount of the mineral filler employed varied from 0 to $40 \mathrm{phr}$. The mechanical and dynamic-mechanical properties of different compositions were evaluated as well as crosslink density, before and after ageing, and the results were compared to those obtained for the unfilled composition. It was found that, either before or after ageing, the amount of mica must not exceed $30 \mathrm{phr}$. It was also concluded that mica is responsible for the protection of natural rubber composites from ageing under the selected conditions. The dynamic mechanical properties obtained from DMTA analysis corroborate the mechanical properties evaluated.
\end{abstract}

Keywords: Natural rubber, mica, physical mechanical properties, and dynamic mechanical properties ageing

\section{Introdução}

As propriedades físicas e mecânicas de elastômeros são alteradas pelo envelhecimento, que causa mudanças na estrutura ou na morfologia destes materiais. Em muitas aplicações, também a longevidade dos constituintes elastoméricos tem importância crítica. É interessante ressaltar a existência de componentes de borracha natural em serviço, à temperatura ambiente, por até 100 anos (um longo recorde não alcançado pelas borrachas sintéticas). $\mathrm{O}$ grau de envelhecimento depende de muitos fatores incluindo tipo de polímero, formulação, geometria do produto em teste, e condições ambientais ${ }^{[1]}$.

O envelhecimento de sistemas elastoméricos é um processo químico complexo que ocorre sob influência do calor, oxigênio, luz, ozônio, tensão mecânica, etc, e resulta em modificações das propriedades químicas e físicas, as quais são dependentes do tempo. Devido a sua importância prática, e também por ser facilmente controlado em condi- ções de laboratório, o envelhecimento causado por ação do calor é o mais estudado.

O termo "envelhecimento" em composições de borracha está relacionado com a cisão molecular, que resulta em cadeias menores e num maior número de terminais de cadeia, e/ou em reticulação, que gera uma estrutura em rede fortemente ligada ${ }^{[2]}$.

As duas reações básicas que levam às modificações da estrutura química do elastômero podem ser exemplificadas pelo seguinte mecanismo radicalar simplificado ${ }^{[3]}$ :

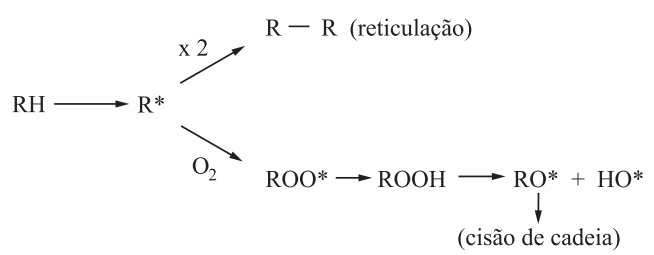

Os dois principais processos de envelhecimento em elastômeros são a oxidação e a ozonólise. A oxidação envolve 
a reação de radicais livres com o oxigênio molecular e é acelerada pelo aumento de temperatura. Os radicais livres podem ser formados pela decomposição de pequenas quantidades de hidroperóxidos, presentes na borracha após processamento. Dependendo do tipo de borracha, essas reações levam tanto à cisão de cadeia quanto ao aumento das ligações cruzadas ${ }^{[4]}$.

É importante considerar que, com a cisão das cadeias, a viscosidade decresce, mas no caso da reticulação, o material torna-se mais rígido. A combinação de ambos os fenômenos resulta na formação de microfissuras, tendo sido já comprovada a presença de alguns produtos oxigenados, mesmo antes que a cisão de cadeia ocorra ${ }^{[3]}$.

As escalas de tempo de interesse para alguns produtos são medidas em décadas e, inevitavelmente a credibilidade nos testes de envelhecimento acelerado torna-se necessária para uma estimativa confiável da resistência desses materiais a este efeito. Os métodos para avaliação das mudanças estruturais que acompanham o envelhecimento dos vulcanizados elastoméricos devem ser melhorados, mas ainda é muito difícil obter uma simulação em laboratório do tempo de serviço a altas taxas de envelhecimento. O problema é tanto maior quanto maiores forem o tempo esperado em serviço e o grau de aceleração aplicado ${ }^{[1]}$.

A borracha natural (NR), por suas características estruturais, é bastante sensível à degradação, e por isso os aditivos incorporados a uma formulação de NR podem apenas minimizar os efeitos de envelhecimento, como no caso dos antioxidantes e antiozonantes, mas não impedi-los ${ }^{[1,5]}$. Além dos componentes necessários a uma formulação vulcanizável de borracha diênica, como no caso da NR, cargas também são usadas, principalmente para o barateamento e/ou aumento do desempenho mecânico do artefato final. Neste trabalho, mica foi a carga escolhida com base nas seguintes propriedades: alta resistência térmica, natureza mineral e estrutura laminar, aliando características de interesse tecnológico como baixo custo e grande abundância no Brasil ${ }^{[6,7]}$.

Este trabalho teve por objetivo estudar o efeito do envelhecimento acelerado em composições vulcanizáveis de borracha natural com mica, com teores de carga variando entre 0 e 40phr. As diferentes composições foram avaliadas, antes e após envelhecimento, quanto às propriedades mecânicas e dinâmico-mecânicas, e também quanto à densidade de ligações cruzadas.

\section{Experimental}

A preparação das composições de borracha natural com mica, a determinação das propriedades mecânicas e dinâmicomecânicas, e da densidade de ligação cruzada estão descritas em trabalho anterior ${ }^{[8]}$.

Todas as composições de borracha natural estudadas foram submetidas ao envelhecimento acelerado em estufa com circulação forçada de ar, a $70{ }^{\circ} \mathrm{C}$, durante 96 horas, de acordo com as normas ASTM D 573 e D 1349. As misturas envelhecidas foram então avaliadas quanto à resistência à tração, dureza, densidade de ligação cruzada e propriedades dinâmico-mecânicas. As espessuras dos corpos-de-prova usadas nos ensaios de envelhecimento foram de $0,6 \mathrm{~cm}$ para dureza, e de $0,2 \mathrm{~cm}$ nos demais testes.

\section{Resultados e Discussão}

Para efeito de comparação, todas as propriedades determinadas após envelhecimento acelerado foram as mesmas dos materiais originais, cujos resultados foram discutidos anteriormente ${ }^{[8]}$.

Os resultados de tração e alongamento na ruptura, antes e após o envelhecimento acelerado, estão apresentados nas Figuras 1 e 2 respectivamente. No caso de tração na ruptura é possível observar que houve um ligeiro aumento na composição com 30phr de mica, antes e após o envelhecimento acelerado. Além disso, os valores encontrados mostram que as condições escolhidas para o envelhecimento levaram a uma pequena redução na resistência à tração e uma redução mais significativa no alongamento na ruptura para todas as composições estudadas, com e sem carga, acima de 30phr de mica. Os dois mecanismos de envelhecimento podem estar atuando, isto é, o do enfraquecimento da matriz elastomérica devido à cisão da cadeia principal da borracha e/ou o da formação de excesso de ligações cruzadas, que também contribui para a diminuição das propriedades analisadas ${ }^{[1,9]}$.

Os resultados de densidade de ligações cruzadas são mostrados na Figura 3. Pode ser visto que o envelhecimento causa o aumento deste parâmetro para todas as composições estudadas. Este aumento pode ser explicado pelas reações de pós-cura e de reticulação oxidativa ${ }^{[9]}$. Para a maioria das aplicações, a densidade de ligações cruzadas deve ser suficiente para manter a integridade mecânica da borracha, de tal forma que ela suporte carga e apresente recuperação após deformação. Porém esta densidade não deve ser elevada, imobilizando as cadeias poliméricas, o que leva a artefatos duros, quebradiços ${ }^{[4]}$.

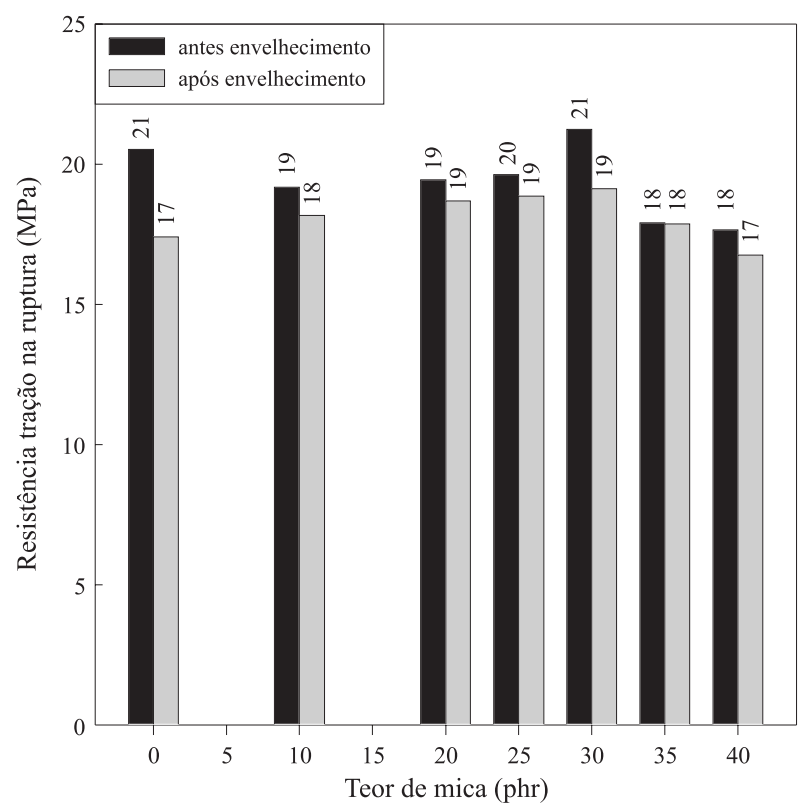

Figura 1. Resistência à tração na ruptura de composições NR/mica antes e após envelhecimento acelerado a $70{ }^{\circ} \mathrm{C} ; 96 \mathrm{~h}$ 


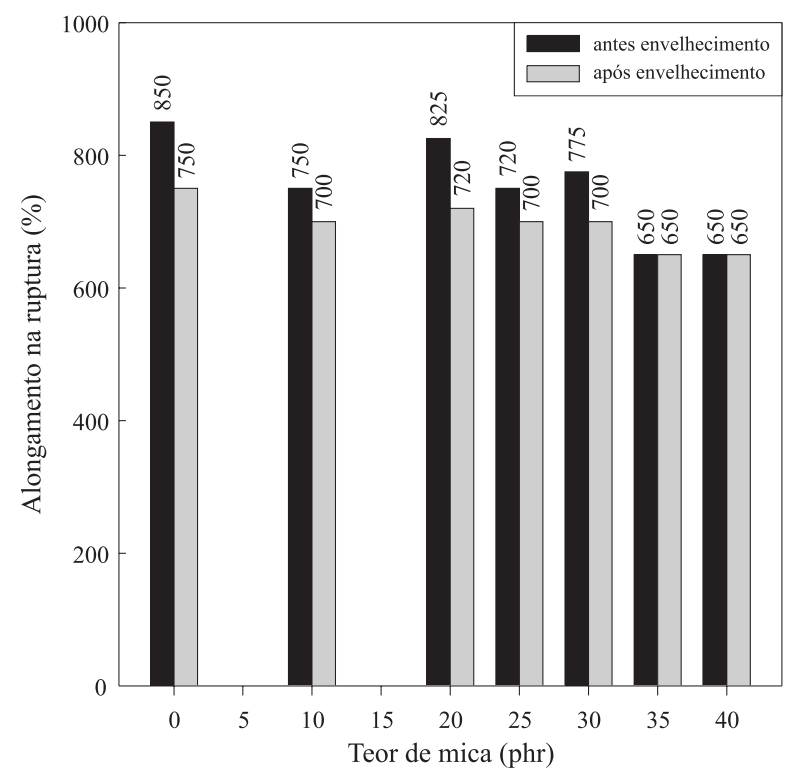

Figura 2. Alongamento na ruptura de composições NR/mica antes e após envelhecimento acelerado a $70{ }^{\circ} \mathrm{C} ; 96 \mathrm{~h}$

Os valores mais elevados de densidade de ligações cruzadas, principalmente para as composições com teores acima de 30 phr de mica, explicam a diminuição da tração e do alongamento na ruptura (Figuras 1 e 2), antes e após envelhecimento acelerado.

Os resultados de módulo e dureza, característicos da rigidez de um artefato, estão apresentados na Tabela 1. Como era de se esperar, a dureza aumenta com a incorporação crescente de carga, antes do envelhecimento acelerado, o que também está relacionado com os valores mais altos de densidade de ligações cruzadas encontrados para as composições NR/mica (Figura

Tabela 1. Dureza e módulo de composições NR/mica antes e após envelhecimento acelerado a $70{ }^{\circ} \mathrm{C} ; 96 \mathrm{~h}$

\begin{tabular}{cccc}
\hline \multirow{2}{*}{$\begin{array}{c}\text { Envelhecimento } \\
\text { acelerado }\end{array}$} & Propriedade & \multicolumn{2}{c}{ Teor de mica (phr) } \\
\cline { 3 - 4 } & 0 & Antes & Após \\
\hline & 10 & 35 & 36 \\
Dureza & 20 & 38 & 40 \\
(Shore A) & 25 & 41 & 43 \\
& 30 & 41 & 44 \\
& 35 & 43 & 46 \\
& 40 & 44 & 48 \\
\hline & 0 & 1,20 & 1,49 \\
& 10 & 1,77 & 1,93 \\
Módulo a 300\% & 20 & 1,98 & 2,38 \\
(MPa) & 25 & 2,11 & 2,27 \\
& 30 & 2,76 & 2,25 \\
& 35 & 2,98 & 2,38 \\
& 40 & 2,94 & 2,78 \\
\hline
\end{tabular}

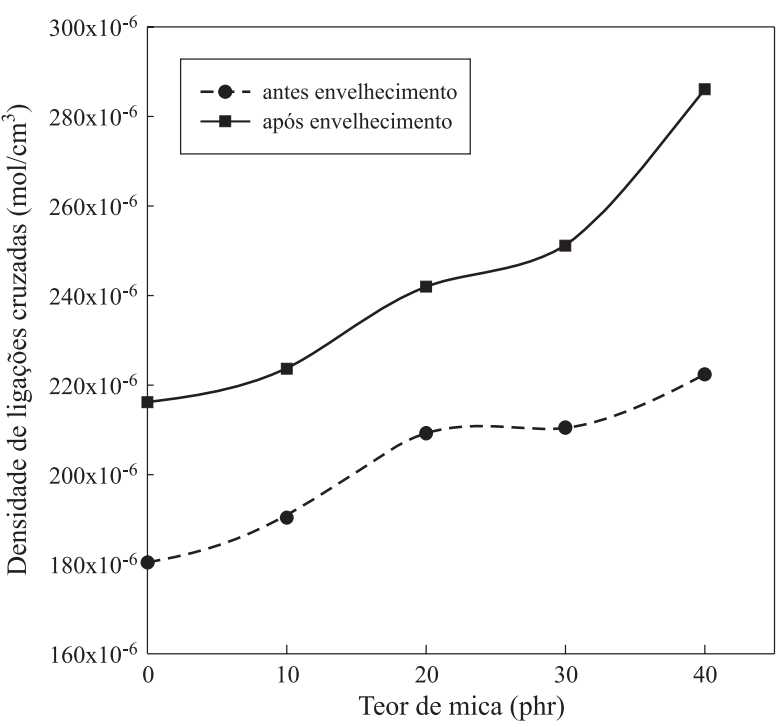

Figura 3. Densidade de ligações cruzadas de composições NR/mica antes e após envelhecimento acelerado a $70{ }^{\circ} \mathrm{C} ; 96 \mathrm{~h}$

3). Ambos os parâmetros apresentaram o mesmo comportamento após envelhecimento. Os valores do módulo a 300\%, após envelhecimento, apresentaram tendência a decréscimo, quando comparado aos valores obtidos antes do envelhecimento, para as composições acima de $20 \mathrm{phr}$ de mica. O decréscimo no valor do módulo após envelhecimento pode ser justificado com base no mecanismo de degradação de borrachas diênicas, que leva à despolimerização, isto é, ao enfraquecimento da matriz elastomérica causado pela cisão de cadeias e pelo aumento do número de ligações cruzadas ${ }^{[3]}$.

As propriedades dinâmicas de composições NR/mica, obtidas por DMTA, estão apresentadas na Tabela 2. Antes do envelhecimento é possível observar que as composições NR/ mica mostram ligeira tendência ao deslocamento da $T_{g}$ para valores inferiores ao da NR, o que indica um certo grau de dispersão da carga na matriz elastomérica, à exceção da que contém 30phr. Para este teor de mica os resultados sugerem algum tipo de interação borracha-carga.

O módulo elástico foi também influenciado pela presença de mica e seu teor, tanto antes quanto após o envelhecimento. Durante o processo de mistura, um tipo de estrutura

Tabela 2. Propriedades dinâmicas de composições NR/mica

\begin{tabular}{|c|c|c|c|c|c|c|}
\hline \multirow{2}{*}{$\begin{array}{c}\text { Envelhecimento } \\
\text { acelerado }\end{array}$} & \multirow{2}{*}{ Propriedade } & \multicolumn{5}{|c|}{ Teor de Mica } \\
\hline & & 0 & 10 & 20 & 30 & 40 \\
\hline \multirow{3}{*}{ Antes } & $\mathbf{T}_{\mathrm{g}}\left({ }^{\circ} \mathbf{C}\right)$ & -52 & -55 & -55 & -52 & -54 \\
\hline & $\operatorname{Tan} \delta$ & 2,13 & 1,98 & 1,95 & 1,76 & 1,73 \\
\hline & $\log \mathbf{E}^{\prime}(\mathbf{P a})^{*}$ & - & - & 5,37 & 5,38 & 5,75 \\
\hline \multirow{3}{*}{ Após } & $\mathbf{T}_{\mathrm{g}}\left({ }^{\circ} \mathbf{C}\right)$ & -54 & -54 & -55 & -54 & -54 \\
\hline & $\operatorname{Tan} \delta$ & 1,85 & 1,83 & 1,80 & 1,77 & 1,70 \\
\hline & $\log \mathbf{E}^{\prime}(\mathbf{P a})^{*}$ & 5,56 & 5,93 & 6,07 & 6,04 & 6,02 \\
\hline
\end{tabular}

* valores a $0{ }^{\circ} \mathrm{C}$ 
elastômero-carga é formado, mais conhecido como borracha ocluída ${ }^{[10,11]}$. Esta estrutura tem grande influência durante a deformação, e caracteriza a carga quanto ao seu carater reforçador ou não. No caso da mica, ela pode ser considerada como semi-reforçadora, por não manter esta interação sob altas deformações, como mostrado nos resultados das propriedades mecânicas, mas sendo responsável pelo aumento das propriedades, quando os resultados são comparados aos da composição sem carga. O teor da mica leva ao aumento da borracha ocluída, causando o aumento de E'. Como a tan $\delta$ é a razão E'/E', com aumento de E' a tan $\delta$ diminui, como pode ser comprovado pelos resultados obtidos antes e após o envelhecimento. Valores mais elevados de E' com a incorporação crescente de carga e portanto menores valores de $\tan \delta$, têm comportamento conforme esperado devido ao tipo de interação carga-elastômero que está ocorrendo.

Após envelhecimento é possível notar o efeito deste na $\mathrm{NR}$, representado pela ligeira diminuição de sua $T_{g}$, enquanto as das composições com mica não sofrem alterações. Estes resultados indicam que as características da mica foram as responsáveis pela resistência ao envelhecimento das composições de borracha natural nas condições escolhidas, principalmente para teores desta carga mineral em torno de $20 \mathrm{phr}$. Os valores mais elevados de E', superiores aos obtidos antes do envelhecimento, estão relacionados não somente ao maior número de ligações cruzadas formados (Figura 3 ), mas sugerem que as interações borracha-carga foram mantidas, e assim pode-se afirmar que as propriedades dinâmico-mecânicas corroboram as propriedades mecânicas estudadas.

\section{Conclusões}

- O desempenho mecânico antes e após envelhecimento acelerado, indica 30phr como limite do teor de mica nas composições de NR.

- Os resultados das propriedades dinâmico-mecânicas sugerem a presença de interações borracha-carga, mesmo após o envelhecimento.

- O mecanismo de envelhecimento predominante nas composições NR/mica é do tipo reticulação oxidativa.

- Os resultados obtidos indicam que as características da mica foram as responsáveis pela resistência ao envelhecimento das composições de NR, nas condições escolhidas.

\section{Agradecimentos}

Os autores agradecem ao Conselho Nacional de Desenvolvimento Científico e Tecnológico (CNPq) e à Fundação Universitária José Bonifácio (FUJB) pelo apoio financeiro, e a Brasilminas Indústria e Comércio Ltda pela doação da mica.

\section{Referências bibliográficas}

1. Brown, R. P; Forrest, M. J. \& Soulagnet, G. - Rapra, 10(2), p.3, (1999).

2. Garbarczyk, M.; Kuhn, W.; Klinowski, J. \& Jurga, S. Polym., 43, p.3169, (2002).

3. Lucas, P.; Baba, M.; Lacoste, J. \& Gardette, J. L. - Polym. Degr. Stab., 76, p.449, (2002).

4. Somers, A. E.; Bastow, T. J.; Burgar, M. I.; Forsyth, M. \& Hill, A. J. - Polym. Degr. Stab., 70, p.31, (2000).

5. Chapman, A. V.\& Porter, M. in Natural Rubber Science and Technology., Roberts, A. D. (ed.) Oxford Scientific Publication, New York, cap 12, (1988).

6. http://mineral.galleries.com.

7. Shell,H. R. - Mica in Encyclopedia of Chemical Technology, Vol 13, Kirk Othmer (ed.) John Willey and Sons Inc. New York, (1965).

8. Escócio, V. A.; Martins, A. F.; Costa, D. M. R; Visconte, L. L. Y. \& Nunes, R. C. R. - Polímeros: Ciência e Tecnologia 13 (2), p.132, (2003).

9. Mathew, N. M. \& De, S. K. - Polym. 24, p.1042, (1983).

10. Mark, J. E., Erman, B. \& Eirich, F. R - Science and Techonology of Rubber, Academic Press, New York, (1994).

11. Chuayjuljit, S.; Imvittaya, A.; Na-Ranong, N. \& Potiyaraj, P. - Journal of Metals, Materials and Minerals 12 (1) p.51, (2002).

Recebido: 20/06/03 Aprovado: 21/09/03 